FORMALIZED MATHEMATICS

Vol. 21, No. 3, Pages 193-205, 2013

DOI: $10.2478 /$ forma-2013-0021
VERSIT $\Lambda$

degruyter.com/view/j/forma

\title{
Object-Free Definition of Categories
}

\author{
Marco Riccardi \\ Via del Pero 102 \\ 54038 Montignoso \\ Italy
}

\begin{abstract}
Summary. Category theory was formalized in Mizar with two different approaches 7, 18 that correspond to those most commonly used 16, 5. Since there is a one-to-one correspondence between objects and identity morphisms, some authors have used an approach that does not refer to objects as elements of the theory, and are usually indicated as object-free category [1] or as arrowsonly category 16. In this article is proposed a new definition of an object-free category, introducing the two properties: left composable and right composable, and a simplification of the notation through a symbol, a binary relation between morphisms, that indicates whether the composition is defined. In the final part we define two functions that allow to switch from the two definitions, with and without objects, and it is shown that their composition produces isomorphic categories.
\end{abstract}

MSC: 18A05 03B35

Keywords: object-free category; correspondence between different approaches to category

MML identifier: CAT_6 version: 8.1.02 5.19.1189

The notation and terminology used in this paper have been introduced in the following articles: 6], 2], 7], 8], [4, [14], [9], [10], 11], 15], 19], [3], 12], 21], [22], [17], 20], and [13].

\section{Yet Another Definition of Category}

We consider category structures which extend 1-sorted structures and are systems

〈a carrier, a composition〉

where the carrier is a set, the composition is a partial function from (the carrier) $\times$ the carrier to the carrier.

(C) 2013 University of Białystok CC-BY-SA License ver. 3.0 or later ISSN 1426-2630(Print), 1898-9934(Online) 
In this paper $\mathscr{C}$ denotes a category structure.

Let us consider $\mathscr{C}$. The functor Mor $\mathscr{C}$ yielding a set is defined by the term

(Def. 1) The carrier of $\mathscr{C}$.

A morphism of $\mathscr{C}$ is an element of Mor $\mathscr{C}$. In the sequel $f, f_{1}, f_{2}, f_{3}$ denote morphisms of $\mathscr{C}$.

Let us consider $\mathscr{C}, f_{1}$, and $f_{2}$. We say that $f_{1}$ and $f_{2}$ are composable if and only if

(Def. 2) $\left\langle f_{1}, f_{2}\right\rangle \in$ dom the composition of $\mathscr{C}$.

We introduce $f_{1} \triangleright f_{2}$ as a synonym of $f_{1}$ and $f_{2}$ are composable.

Assume $f_{1} \triangleright f_{2}$. The functor $f_{1} \circ f_{2}$ yielding a morphism of $\mathscr{C}$ is defined by the term

(Def. 3) (The composition of $\mathscr{C})\left(f_{1}, f_{2}\right)$.

Let us consider $f$. We say that $f$ is left identity if and only if

(Def. 4) Let us consider a morphism $f_{1}$ of $\mathscr{C}$. If $f \triangleright f_{1}$, then $f \circ f_{1}=f_{1}$.

We say that $f$ is right identity if and only if

(Def. 5) Let us consider a morphism $f_{1}$ of $\mathscr{C}$. If $f_{1} \triangleright f$, then $f_{1} \circ f=f_{1}$.

We say that $\mathscr{C}$ has left identities if and only if

(Def. 6) Let us consider a morphism $f_{1}$ of $\mathscr{C}$. Suppose $f_{1} \in$ the carrier of $\mathscr{C}$. Then there exists a morphism $f$ of $\mathscr{C}$ such that

(i) $f \triangleright f_{1}$, and

(ii) $f$ is left identity.

We say that $\mathscr{C}$ has right identities if and only if

(Def. 7) Let us consider a morphism $f_{1}$ of $\mathscr{C}$. Suppose $f_{1} \in$ the carrier of $\mathscr{C}$. Then there exists a morphism $f$ of $\mathscr{C}$ such that

(i) $f_{1} \triangleright f$, and

(ii) $f$ is right identity.

We say that $\mathscr{C}$ is left composable if and only if

(Def. 8) Let us consider morphisms $f, f_{1}, f_{2}$ of $\mathscr{C}$. Suppose $f_{1} \triangleright f_{2}$. Then $f_{1} \circ f_{2} \triangleright f$ if and only if $f_{2} \triangleright f$.

We say that $\mathscr{C}$ is right composable if and only if

(Def. 9) Let us consider morphisms $f, f_{1}, f_{2}$ of $\mathscr{C}$. Suppose $f_{1} \triangleright f_{2}$. Then $f \triangleright f_{1} \circ f_{2}$ if and only if $f \triangleright f_{1}$.

We say that $\mathscr{C}$ is associative if and only if

(Def. 10) Let us consider morphisms $f_{1}, f_{2}, f_{3}$ of $\mathscr{C}$. Suppose

(i) $f_{1} \triangleright f_{2}$, and

(ii) $f_{2} \triangleright f_{3}$, and

(iii) $f_{1} \circ f_{2} \triangleright f_{3}$, and 
(iv) $f_{1} \triangleright f_{2} \circ f_{3}$.

Then $f_{1} \circ\left(f_{2} \circ f_{3}\right)=\left(f_{1} \circ f_{2}\right) \circ f_{3}$.

We say that $\mathscr{C}$ is composable if and only if

(Def. 11) $\mathscr{C}$ is left and right composable.

We say that $\mathscr{C}$ has identities if and only if

(Def. 12) $\mathscr{C}$ has left and right identities.

Let $X$ be a set and $f$ be a partial function from $X \times X$ to $X$. Note that the functor $\curvearrowleft f$ yields a partial function from $X \times X$ to $X$. Let us consider $\mathscr{C}$. The functor $\mathscr{C}^{\text {op }}$ yielding a strict category structure is defined by the term

(Def. 13) 〈the carrier of $\mathscr{C}, \curvearrowleft$ the composition of $\mathscr{C}\rangle$.

Now we state the proposition:

(1) If $\mathscr{C}$ is empty, then $f_{1} \not f_{2}$.

In this paper $g_{1}, g_{2}$ denote morphisms of $\mathscr{C}^{\mathrm{op}}$.

Now we state the propositions:

(2) If $f_{1}=g_{1}$ and $f_{2}=g_{2}$, then $f_{1} \triangleright f_{2}$ iff $g_{2} \triangleright g_{1}$.

(3) If $f_{1}=g_{1}$ and $f_{2}=g_{2}$ and $f_{1} \triangleright f_{2}$, then $f_{1} \circ f_{2}=g_{2} \circ g_{1}$.

(4) $\mathscr{C}$ is left composable if and only if $\mathscr{C}^{\text {op }}$ is right composable. The theorem is a consequence of (3). Proof: For every morphisms $f, f_{1}, f_{2}$ of $\mathscr{C}$ such that $f_{1} \triangleright f_{2}$ holds $f_{1} \circ f_{2} \triangleright f$ iff $f_{2} \triangleright f$ by [11, (42)].

(5) $\mathscr{C}$ is right composable if and only if $\mathscr{C}^{\text {op }}$ is left composable. The theorem is a consequence of (3). Proof: For every morphisms $f, f_{1}, f_{2}$ of $\mathscr{C}$ such that $f_{1} \triangleright f_{2}$ holds $f \triangleright f_{1} \circ f_{2}$ iff $f \triangleright f_{1}$ by [11, (42)].

(6) $\mathscr{C}$ has left identities if and only if $\mathscr{C}^{\text {op }}$ has right identities. The theorem is a consequence of (3). Proof: For every morphism $f_{1}$ of $\mathscr{C}$ such that $f_{1} \in$ the carrier of $\mathscr{C}$ there exists a morphism $f$ of $\mathscr{C}$ such that $f \triangleright f_{1}$ and $f$ is left identity by [11, (42)].

(7) $\mathscr{C}$ has right identities if and only if $\mathscr{C}^{\text {op }}$ has left identities. The theorem is a consequence of (3). Proof: For every morphism $f_{1}$ of $\mathscr{C}$ such that $f_{1} \in$ the carrier of $\mathscr{C}$ there exists a morphism $f$ of $\mathscr{C}$ such that $f_{1} \triangleright f$ and $f$ is right identity by [11, (42)].

(8) $\mathscr{C}$ is associative if and only if $\mathscr{C}^{\mathrm{op}}$ is associative. The theorem is a consequence of (3). Proof: For every morphisms $f_{1}, f_{2}, f_{3}$ of $\mathscr{C}$ such that $f_{1} \triangleright f_{2}$ and $f_{2} \triangleright f_{3}$ and $f_{1} \circ f_{2} \triangleright f_{3}$ and $f_{1} \triangleright f_{2} \circ f_{3}$ holds $f_{1} \circ\left(f_{2} \circ f_{3}\right)=\left(f_{1} \circ f_{2}\right) \circ f_{3}$ by [11, (42)].

Note that there exists a category structure which is composable and associative and has left identities and has not right identities and there exists a category structure which is composable and associative and has right identities and has not left identities and there exists a category structure which is non left composable, right composable, and associative and has identities and there 
exists a category structure which is left composable, non right composable, and associative and has identities and there exists a category structure which is non associative and composable and has identities and there exists a category structure which is empty and every category structure which is empty is also left and right composable and associative and has also left and right identities and there exists a category structure which is strict, left and right composable, and associative and has left and right identities and there exists a category structure which is strict, composable, and associative and has identities.

A category is a composable associative category structure with identities. Let us consider $\mathscr{C}$ and $f$. We say that $f$ is identity if and only if

(Def. 14) $f$ is left and right identity.

Now we state the propositions:

(9) If $\mathscr{C}$ has identities, then $f$ is left identity iff $f$ is right identity. Proof: For every morphism $f_{1}$ of $\mathscr{C}$ such that $f \triangleright f_{1}$ holds $f \circ f_{1}=f_{1}$.

(10) If $\mathscr{C}$ is empty, then $f$ is identity.

(11) Let us consider morphisms $g_{1}, g_{2}$ of the category structure of $\mathscr{C}$. Suppose

(i) $f_{1}=g_{1}$, and

(ii) $f_{2}=g_{2}$, and

(iii) $f_{1} \triangleright f_{2}$.

Then $f_{1} \circ f_{2}=g_{1} \circ g_{2}$.

(12) $\mathscr{C}$ is left composable if and only if the category structure of $\mathscr{C}$ is left composable. The theorem is a consequence of (11). Proof: For every morphisms $f, f_{1}, f_{2}$ of $\mathscr{C}$ such that $f_{1} \triangleright f_{2}$ holds $f_{1} \circ f_{2} \triangleright f$ iff $f_{2} \triangleright f$.

(13) $\mathscr{C}$ is right composable if and only if the category structure of $\mathscr{C}$ is right composable. The theorem is a consequence of (11). Proof: For every morphisms $f, f_{1}, f_{2}$ of $\mathscr{C}$ such that $f_{1} \triangleright f_{2}$ holds $f \triangleright f_{1} \circ f_{2}$ iff $f \triangleright f_{1}$.

(14) $\mathscr{C}$ is composable if and only if the category structure of $\mathscr{C}$ is composable.

(15) $\mathscr{C}$ is associative if and only if the category structure of $\mathscr{C}$ is associative. The theorem is a consequence of (11). Proof: For every morphisms $f_{1}$, $f_{2}, f_{3}$ of $\mathscr{C}$ such that $f_{1} \triangleright f_{2}$ and $f_{2} \triangleright f_{3}$ and $f_{1} \circ f_{2} \triangleright f_{3}$ and $f_{1} \triangleright f_{2} \circ f_{3}$ holds $f_{1} \circ\left(f_{2} \circ f_{3}\right)=\left(f_{1} \circ f_{2}\right) \circ f_{3}$.

(16) Let us consider a morphism $g$ of the category structure of $\mathscr{C}$. If $f=g$, then $f$ is left identity iff $g$ is left identity. The theorem is a consequence of (11). PRoOF: For every morphism $f_{2}$ of $\mathscr{C}$ such that $f \triangleright f_{2}$ holds $f \circ f_{2}=f_{2}$.

(17) $\mathscr{C}$ has left identities if and only if the category structure of $\mathscr{C}$ has left identities. The theorem is a consequence of (16). Proof: For every morphism $f_{1}$ of $\mathscr{C}$ such that $f_{1} \in$ the carrier of $\mathscr{C}$ there exists a morphism $f$ of $\mathscr{C}$ such that $f \triangleright f_{1}$ and $f$ is left identity. 
(18) Let us consider a morphism $g$ of the category structure of $\mathscr{C}$. If $f=g$, then $f$ is right identity iff $g$ is right identity. The theorem is a consequence of (11). Proof: For every morphism $f_{1}$ of $\mathscr{C}$ such that $f_{1} \triangleright f$ holds $f_{1} \circ f=$ $f_{1}$.

(19) $\mathscr{C}$ has right identities if and only if the category structure of $\mathscr{C}$ has right identities. The theorem is a consequence of (18). Proof: For every morphism $f_{1}$ of $\mathscr{C}$ such that $f_{1} \in$ the carrier of $\mathscr{C}$ there exists a morphism $f$ of $\mathscr{C}$ such that $f_{1} \triangleright f$ and $f$ is right identity.

(20) $\mathscr{C}$ has identities if and only if the category structure of $\mathscr{C}$ has identities.

Let us consider $\mathscr{C}$. We say that $\mathscr{C}$ is discrete if and only if

(Def. 15) Every morphism of $\mathscr{C}$ is identity.

One can verify that there exists a category structure which is strict, empty, discrete, composable, and associative and has identities.

Now we state the proposition:

(21) Let us consider a discrete category structure $\mathscr{C}$ and morphisms $f_{1}, f_{2}$ of $\mathscr{C}$. If $f_{1} \triangleright f_{2}$, then $f_{1}=f_{2}$ and $f_{1} \circ f_{2}=f_{2}$.

Observe that every category structure which is discrete is also composable and associative.

Let $X$ be a set. The discrete category of $X$ yielding a strict discrete category is defined by

(Def. 16) The carrier of $i t=X$.

Note that there exists a category which is strict and there exists a category which is strict and empty and there exists a category which is strict and non empty.

Let us consider $\mathscr{C}$. The functor $\mathrm{Ob} \mathscr{C}$ yielding a subset of Mor $\mathscr{C}$ is defined by the term

(Def. 17) $\{f$, where $f$ is a morphism of $\mathscr{C}: f$ is identity and $f \in \operatorname{Mor} \mathscr{C}\}$.

An object of $\mathscr{C}$ is an element of $\mathrm{Ob} \mathscr{C}$. Let $\mathscr{C}$ be a non empty category structure with identities. Let us observe that $\mathrm{Ob} \mathscr{C}$ is non empty.

Now we state the propositions:

(22) Let us consider a non empty category structure $\mathscr{C}$ with identities and a morphism $f$ of $\mathscr{C}$. Then $f$ is identity if and only if $f$ is an object of $\mathscr{C}$.

(23) Let us consider a non empty category structure $\mathscr{C}$ with identities, morphisms $f, f_{1}$ of $\mathscr{C}$, and an object $o$ of $\mathscr{C}$. Suppose $f=o$. Then

(i) if $f \triangleright f_{1}$, then $f \circ f_{1}=f_{1}$, and

(ii) if $f_{1} \triangleright f$, then $f_{1} \circ f=f_{1}$, and

(iii) $f \triangleright f$.

The theorem is a consequence of (22). 
(24) Let us consider a non empty category structure $\mathscr{C}$ with identities and a morphism $f$ of $\mathscr{C}$. If $f$ is identity, then $f \triangleright f$. The theorem is a consequence of $(22)$ and (23).

(25) Let us consider category structures $\mathscr{C}_{1}, \mathscr{C}_{2}$ with identities.

Suppose the category structure of $\mathscr{C}_{1}=$ the category structure of $\mathscr{C}_{2}$. Let us consider a morphism $f_{1}$ of $\mathscr{C}_{1}$ and a morphism $f_{2}$ of $\mathscr{C}_{2}$. If $f_{1}=f_{2}$, then $f_{1}$ is identity iff $f_{2}$ is identity. Proof: For every morphism $f$ of $\mathscr{C}_{1}$ such that $f_{1} \triangleright f$ holds $f_{1} \circ f=f$. For every morphism $f$ of $\mathscr{C}_{1}$ such that $f \triangleright f_{1}$ holds $f \circ f_{1}=f$.

Let $\mathscr{C}$ be a composable category structure with identities and $f$ be a morphism of $\mathscr{C}$. The functor dom $f$ yielding an object of $\mathscr{C}$ is defined by

(Def. 18) (i) there exists a morphism $f_{1}$ of $\mathscr{C}$ such that it $=f_{1}$ and $f \triangleright f_{1}$ and $f_{1}$ is identity, if $\mathscr{C}$ is not empty,

(ii) $i t=$ the object of $\mathscr{C}$, otherwise.

The functor $\operatorname{cod} f$ yielding an object of $\mathscr{C}$ is defined by

(Def. 19) (i) there exists a morphism $f_{1}$ of $\mathscr{C}$ such that it $=f_{1}$ and $f_{1} \triangleright f$ and $f_{1}$ is identity, if $\mathscr{C}$ is not empty,

(ii) $i t=$ the object of $\mathscr{C}$, otherwise.

Let us consider a composable category structure $\mathscr{C}$ with identities and morphisms $f, f_{1}$ of $\mathscr{C}$. Now we state the propositions:

(26) If $f \triangleright f_{1}$ and $f_{1}$ is identity, then $\operatorname{dom} f=f_{1}$.

(27) If $f_{1} \triangleright f$ and $f_{1}$ is identity, then $\operatorname{cod} f=f_{1}$.

Let $\mathscr{C}$ be category structure with identities and $o$ be an object of $\mathscr{C}$. The functor id- $o$ yielding a morphism of $\mathscr{C}$ is defined by the term

(Def. 20) $o$.

Let $\mathscr{C}, \mathscr{D}$ be category structures. A functor from $\mathscr{C}$ to $\mathscr{D}$ is a function from $\mathscr{C}$ into $\mathscr{D}$. In the sequel $\mathscr{C}, \mathscr{D}, \mathscr{E}$ denote category structures with identities, $\mathcal{F}$ denotes a functor from $\mathscr{C}$ to $\mathscr{D}, \mathcal{G}$ denotes a functor from $\mathscr{D}$ to $\mathscr{E}$, and $f$ denotes a morphism of $\mathscr{C}$.

Let us consider $\mathscr{C}, \mathscr{D}, \mathcal{F}$, and $f$. The functor $\mathcal{F}(f)$ yielding a morphism of $\mathscr{D}$ is defined by the term

(Def. 21) $\begin{cases}\mathcal{F}(f), & \text { if } \mathscr{C} \text { is not empty }, \\ \text { The object of } \mathscr{D}, & \text { otherwise. }\end{cases}$

We say that $\mathcal{F}$ preserves identity if and only if

(Def. 22) Let us consider a morphism $f$ of $\mathscr{C}$. If $f$ is identity, then $\mathcal{F}(f)$ is identity. We say that $\mathcal{F}$ is multiplicative if and only if

(Def. 23) Let us consider morphisms $f_{1}, f_{2}$ of $\mathscr{C}$. Suppose $f_{1} \triangleright f_{2}$. Then

(i) $\mathcal{F}\left(f_{1}\right) \triangleright \mathcal{F}\left(f_{2}\right)$, and

(ii) $\mathcal{F}\left(f_{1} \circ f_{2}\right)=\mathcal{F}\left(f_{1}\right) \circ \mathcal{F}\left(f_{2}\right)$. 
We say that $\mathcal{F}$ is anti-multiplicative if and only if

(Def. 24) Let us consider morphisms $f_{1}, f_{2}$ of $\mathscr{C}$. Suppose $f_{1} \triangleright f_{2}$. Then

(i) $\mathcal{F}\left(f_{2}\right) \triangleright \mathcal{F}\left(f_{1}\right)$, and

(ii) $\mathcal{F}\left(f_{1} \circ f_{2}\right)=\mathcal{F}\left(f_{2}\right) \circ \mathcal{F}\left(f_{1}\right)$.

Note that there exists a functor from $\mathscr{C}$ to $\mathscr{D}$ which preserves identity.

Let $\mathscr{C}$ be an empty category structure with identities and $\mathscr{D}$ be category structure with identities. Note that there exists a functor from $\mathscr{C}$ to $\mathscr{D}$ which is multiplicative and anti-multiplicative preserves identity.

Let $\mathscr{C}$ be category structure with identities and $\mathscr{D}$ be a non empty category structure with identities. Let us observe that there exists a functor from $\mathscr{C}$ to $\mathscr{D}$ which is multiplicative and anti-multiplicative preserves identity.

Now we state the propositions:

(28) There exist categories $\mathscr{C}, \mathscr{D}$ and there exists a functor $\mathcal{F}$ from $\mathscr{C}$ to $\mathscr{D}$ such that $\mathcal{F}$ is multiplicative and $\mathcal{F}$ does not preserve identity. The theorem is a consequence of (22). Proof: Set $\mathscr{C}=$ the non empty category. Reconsider $X=\{0,1\}$ as a set. Set $c_{4}=\{\langle\langle 0,0\rangle, 0\rangle,\langle\langle 1,1\rangle, 1\rangle\} \cup\{\langle\langle 0$, $1\rangle, 1\rangle,\langle\langle 1,0\rangle, 1\rangle\}$. For every element $x, x \in c_{4}$ iff $x=\langle\langle 0,0\rangle, 0\rangle$ or $x=\langle\langle 1,1\rangle, 1\rangle$ or $x=\langle\langle 0,1\rangle, 1\rangle$ or $x=\langle\langle 1,0\rangle, 1\rangle$. For every elements $x, y_{1}, y_{2}$ such that $\left\langle x, y_{1}\right\rangle,\left\langle x, y_{2}\right\rangle \in c_{4}$ holds $y_{1}=y_{2}$. For every element $x$ such that $x \in c_{4}$ holds $x \in(X \times X) \times X$. Set $\mathscr{D}=\left\langle X, c_{4}\right\rangle$. For every morphisms $f_{1}, f_{2}$ of $\mathscr{D}$ such that $f_{1} \triangleright f_{2}$ holds $f_{1}=0$ and $f_{2}=0$ and $f_{1} \circ f_{2}=0$ or $f_{1}=1$ and $f_{2}=1$ and $f_{1} \circ f_{2}=1$ or $f_{1}=0$ and $f_{2}=1$ and $f_{1} \circ f_{2}=1$ or $f_{1}=1$ and $f_{2}=0$ and $f_{1} \circ f_{2}=1$ by [9, (1)]. For every morphisms $f_{1}, f_{2}$ of $\mathscr{D}, f_{1} \triangleright f_{2}$ by [9, (1)]. For every morphism $f_{1}$ of $\mathscr{D}$ such that $f_{1} \in$ the carrier of $\mathscr{D}$ there exists a morphism $f$ of $\mathscr{D}$ such that $f \triangleright f_{1}$ and $f$ is left identity. For every morphism $f_{1}$ of $\mathscr{D}$ such that $f_{1} \in$ the carrier of $\mathscr{D}$ there exists a morphism $f$ of $\mathscr{D}$ such that $f_{1} \triangleright f$ and $f$ is right identity. For every morphisms $f_{1}, f_{2}, f_{3}$ of $\mathscr{D}$ such that $f_{1} \triangleright f_{2}$ and $f_{2} \triangleright f_{3}$ and $f_{1} \circ f_{2} \triangleright f_{3}$ and $f_{1} \triangleright f_{2} \circ f_{3}$ holds $f_{1} \circ\left(f_{2} \circ f_{3}\right)=\left(f_{1} \circ f_{2}\right) \circ f_{3}$. Reconsider $d_{1}=1$ as a morphism of $\mathscr{D}$. Define $\mathcal{H}$ (element) $=d_{1}$. Consider $\mathcal{F}$ being a function from the carrier of $\mathscr{C}$ into the carrier of $\mathscr{D}$ such that for every element $x$ such that $x \in$ the carrier of $\mathscr{C}$ holds $\mathcal{F}(x)=\mathcal{H}(x)$ from [10, Sch. 2]. For every morphisms $f_{1}, f_{2}$ of $\mathscr{C}$ such that $f_{1} \triangleright f_{2}$ holds $\mathcal{F}\left(f_{1}\right) \triangleright \mathcal{F}\left(f_{2}\right)$ and $\mathcal{F}\left(f_{1} \circ f_{2}\right)=\mathcal{F}\left(f_{1}\right) \circ \mathcal{F}\left(f_{2}\right)$. There exists a morphism $f$ of $\mathscr{C}$ such that $f$ is identity and $\mathcal{F}(f)$ is not identity.

(29) Suppose $\mathscr{C}$ is not empty and $\mathscr{D}$ is empty. Then there exists no a functor $\mathcal{F}$ from $\mathscr{C}$ to $\mathscr{D}$ such that $\mathcal{F}$ is multiplicative or $\mathcal{F}$ is anti-multiplicative. The theorem is a consequence of (23).

(30) There exist categories $\mathscr{C}, \mathscr{D}$ and there exists a functor $\mathcal{F}$ from $\mathscr{C}$ to $\mathscr{D}$ such that $\mathcal{F}$ is not multiplicative and $\mathcal{F}$ preserves identity. The theorem is a consequence of (29). 
Let us consider $\mathscr{C}, \mathscr{D}$, and $\mathcal{F}$. We say that $\mathcal{F}$ is covariant if and only if

(i) $\mathcal{F}$ preserves identity, and

(ii) $\mathcal{F}$ is multiplicative.

We say that $\mathcal{F}$ is contravariant if and only if

(Def. 26) (i) $\mathcal{F}$ preserves identity, and

(ii) $\mathcal{F}$ is anti-multiplicative.

Let $\mathscr{C}$ be an empty category structure with identities and $\mathscr{D}$ be category structure with identities. One can check that there exists a functor from $\mathscr{C}$ to $\mathscr{D}$ which is covariant and contravariant.

Let $\mathscr{C}$ be category structure with identities and $\mathscr{D}$ be a non empty category structure with identities. Observe that there exists a functor from $\mathscr{C}$ to $\mathscr{D}$ which is covariant and contravariant.

Now we state the proposition:

(31) Suppose $\mathscr{C}$ is not empty and $\mathscr{D}$ is empty. Then there exists no a functor $\mathcal{F}$ from $\mathscr{C}$ to $\mathscr{D}$ such that $\mathcal{F}$ is covariant or $\mathcal{F}$ is contravariant.

Let $\mathscr{C}, \mathscr{D}$ be non empty category structures with identities, $\mathcal{F}$ be a covariant functor from $\mathscr{C}$ to $\mathscr{D}$, and $f$ be an object of $\mathscr{C}$. Observe that the functor $\mathcal{F}(f)$ yields an object of $\mathscr{D}$. Now we state the propositions:

(32) Let us consider non empty composable category structures $\mathscr{C}, \mathscr{D}$ with identities, a covariant functor $\mathcal{F}$ from $\mathscr{C}$ to $\mathscr{D}$, and a morphism $f$ of $\mathscr{C}$. Then

(i) $\mathcal{F}(\operatorname{dom} f)=\operatorname{dom}(\mathcal{F}(f))$, and

(ii) $\mathcal{F}(\operatorname{cod} f)=\operatorname{cod}(\mathcal{F}(f))$.

The theorem is a consequence of (22).

(33) Let us consider non empty composable category structures $\mathscr{C}, \mathscr{D}$ with identities, a covariant functor $\mathcal{F}$ from $\mathscr{C}$ to $\mathscr{D}$, and an object $o$ of $\mathscr{C}$. Then $\mathcal{F}($ id- $o)=\operatorname{id}-(\mathcal{F}(o))$.

Let us consider $\mathscr{C}, \mathscr{D}, \mathscr{E}, \mathcal{F}$, and $\mathcal{G}$. Assume $\mathcal{F}$ is covariant or $\mathcal{F}$ is contravariant and $\mathcal{G}$ is covariant or $\mathcal{G}$ is contravariant. The functor $\mathcal{G} \circ \mathcal{F}$ yielding a functor from $\mathscr{C}$ to $\mathscr{E}$ is defined by the term

(Def. 27) $\mathcal{F} \cdot \mathcal{G}$.

Now we state the propositions:

(34) Suppose $\mathcal{F}$ is covariant and $\mathcal{G}$ is covariant and $\mathscr{C}$ is not empty. Then $(\mathcal{G} \circ \mathcal{F})(f)=\mathcal{G}(\mathcal{F}(f))$. The theorem is a consequence of $(29)$.

(35) If $\mathcal{F}$ is covariant and $\mathcal{G}$ is covariant, then $\mathcal{G} \circ \mathcal{F}$ is covariant. The theorem is a consequence of (34), (22), and (10). Proof: Set $\mathcal{G}_{1}=\mathcal{G} \circ \mathcal{F}$. For every morphism $f$ of $\mathscr{C}$ such that $f$ is identity holds $\mathcal{G}_{1}(f)$ is identity. For every morphisms $f_{1}, f_{2}$ of $\mathscr{C}$ such that $f_{1} \triangleright f_{2}$ holds $\mathcal{G}_{1}\left(f_{1}\right) \triangleright \mathcal{G}_{1}\left(f_{2}\right)$ and $\mathcal{G}_{1}\left(f_{1} \circ f_{2}\right)=\mathcal{G}_{1}\left(f_{1}\right) \circ \mathcal{G}_{1}\left(f_{2}\right)$. 
Let us consider $\mathscr{C}$. Note that the functor $\operatorname{id}_{\mathscr{C}}$ yields a functor from $\mathscr{C}$ to $\mathscr{C}$. Let us observe that $\operatorname{id}_{\mathscr{C}}$ is covariant.

Let us consider $\mathscr{D}$. We say that $\mathscr{C}$ and $\mathscr{D}$ are isomorphic if and only if

(Def. 28) There exists a functor $\mathcal{F}$ from $\mathscr{C}$ to $\mathscr{D}$ and there exists a functor $\mathcal{G}$ from $\mathscr{D}$ to $\mathscr{C}$ such that $\mathcal{F}$ is covariant and $\mathcal{G}$ is covariant and $\mathcal{G} \circ \mathcal{F}=\operatorname{id}_{\mathscr{C}}$ and $\mathcal{F} \circ \mathcal{G}=\mathrm{id}_{\mathscr{D}}$.

Note that the predicate is reflexive and symmetric.

We introduce $\mathscr{C} \cong \mathscr{D}$ as a synonym of $\mathscr{C}$ and $\mathscr{D}$ are isomorphic.

\section{Transform a Category in the Other}

Let $\mathscr{C}$ be a category structure. The functor CompMap $\mathscr{C}$ yielding a partial function from Mor $\mathscr{C} \times \operatorname{Mor} \mathscr{C}$ to Mor $\mathscr{C}$ is defined by the term

(Def. 29) The composition of $\mathscr{C}$.

Let $\mathscr{C}$ be a composable category structure with identities. The functors: SourceMap $\mathscr{C}$ and TargetMap $\mathscr{C}$ yielding functions from Mor $\mathscr{C}$ into $\mathrm{Ob} \mathscr{C}$ are defined by conditions, respectively.

(Def. 30) (i) for every element $f$ of Mor $\mathscr{C}$, $(\operatorname{SourceMap} \mathscr{C})(f)=\operatorname{dom} f$, if $\mathscr{C}$ is not empty,

(ii) SourceMap $\mathscr{C}=\emptyset$, otherwise.

(Def. 31) (i) for every element $f$ of $\operatorname{Mor} \mathscr{C},(\operatorname{TargetMap} \mathscr{C})(f)=\operatorname{cod} f$, if $\mathscr{C}$ is not empty,

(ii) TargetMap $\mathscr{C}=\emptyset$, otherwise.

Let $\mathscr{C}$ be category structure with identities. The functor IdMap $\mathscr{C}$ yielding a function from $\mathrm{Ob} \mathscr{C}$ into Mor $\mathscr{C}$ is defined by

(Def. 32) (i) for every element $o$ of $\mathrm{Ob} \mathscr{C}, i t(o)=\mathrm{id}-o$, if $\mathscr{C}$ is not empty,

(ii) $i t=\emptyset$, otherwise.

Now we state the propositions:

(36) Let us consider a non empty composable category structure $\mathscr{C}$ with identities and elements $f, g$ of Mor $\mathscr{C}$. Then $\langle g, f\rangle \in \operatorname{dom}$ CompMap $\mathscr{C}$ if and only if $(\operatorname{SourceMap} \mathscr{C})(g)=($ TargetMap $\mathscr{C})(f)$.

(37) Let us consider a composable category structure $\mathscr{C}$ with identities and elements $f, g$ of Mor $\mathscr{C}$. Suppose $($ SourceMap $\mathscr{C})(g)=($ TargetMap $\mathscr{C})(f)$. Then

(i) $($ SourceMap $\mathscr{C})((\operatorname{CompMap} \mathscr{C})(g, f))=(\operatorname{SourceMap} \mathscr{C})(f)$, and

(ii) $($ TargetMap $\mathscr{C})((\operatorname{CompMap} \mathscr{C})(g, f))=(\operatorname{TargetMap} \mathscr{C})(g)$.

The theorem is a consequence of (36). 
(38) Let us consider a composable associative category structure $\mathscr{C}$ with identities and elements $f, g, h$ of Mor $\mathscr{C}$. Suppose

(i) $(\operatorname{SourceMap} \mathscr{C})(h)=(\operatorname{TargetMap} \mathscr{C})(g)$, and

(ii) $(\operatorname{SourceMap} \mathscr{C})(g)=(\operatorname{TargetMap} \mathscr{C})(f)$.

Then $($ CompMap $\mathscr{C})(h,($ CompMap $\mathscr{C})(g, f))=($ CompMap $\mathscr{C})(($ CompMap $\mathscr{C})(h, g), f)$. The theorem is a consequence of $(36)$.

(39) Let us consider a composable category structure $\mathscr{C}$ with identities and an element $b$ of $\mathrm{Ob} \mathscr{C}$. Then

(i) $($ SourceMap $\mathscr{C})(\operatorname{IdMap} \mathscr{C}(b))=b$, and

(ii) $($ TargetMap $\mathscr{C})(\operatorname{IdMap} \mathscr{C}(b))=b$, and

(iii) for every element $f$ of Mor $\mathscr{C}$ such that (TargetMap $\mathscr{C})(f)=b$ holds $($ CompMap $\mathscr{C})(\operatorname{IdMap} \mathscr{C}(b), f)=f$, and

(iv) for every element $g$ of Mor $\mathscr{C}$ such that $(\operatorname{SourceMap} \mathscr{C})(g)=b$ holds $($ CompMap $\mathscr{C})(g, \operatorname{IdMap} \mathscr{C}(b))=g$.

The theorem is a consequence of (22) and (36).

A category defined in [7], to avoid confusion, is called an object-category.

Let $\mathscr{C}$ be a non empty category. The functor $\operatorname{Alter}(\mathscr{C})$ yielding a strict object-category is defined by the term

(Def. 33) 〈Ob $\mathscr{C}$, Mor $\mathscr{C}$, SourceMap $\mathscr{C}$, TargetMap $\mathscr{C}$, CompMap $\mathscr{C}\rangle$.

Let $\mathscr{A}$ be an object-category. The functor alter $\mathscr{A}$ yielding a strict category is defined by the term

(Def. 34) 〈the carrier' of $\mathscr{A},($ the composition of $\mathscr{A})\rangle$.

Observe that alter $\mathscr{A}$ is non empty.

Now we state the propositions:

(40) Let us consider an object-category $\mathscr{A}$, morphisms $a_{1}, a_{2}$ of $\mathscr{A}$, and morphisms $f_{1}, f_{2}$ of alter $\mathscr{A}$. Suppose

(i) $a_{1}=f_{1}$, and

(ii) $a_{2}=f_{2}$, and

(iii) $\left\langle a_{1}, a_{2}\right\rangle \in$ dom the composition of $\mathscr{A}$.

Then $a_{1} \circ a_{2}=f_{1} \circ f_{2}$.

(41) Let us consider an object-category $\mathscr{A}$ and a morphism $f$ of alter $\mathscr{A}$. Then $f$ is identity if and only if there exists an object $o$ of $\mathscr{A}$ such that $f=\mathrm{id}_{o}$. The theorem is a consequence of $(22),(23)$, and (40). Proof: For every morphism $f_{1}$ of alter $\mathscr{A}$ such that $f \triangleright f_{1}$ holds $f \circ f_{1}=f_{1}$ by [7, (15), (21)]. For every morphism $f_{1}$ of alter $\mathscr{A}$ such that $f_{1} \triangleright f$ holds $f_{1} \circ f=f_{1}$ by [7, (15), (22)]. 
(42) Let us consider object-categories $\mathscr{A}, \mathscr{B}$. Then every functor from $\mathscr{A}$ to $\mathscr{B}$ is a covariant functor from alter $\mathscr{A}$ to alter $\mathscr{B}$. The theorem is a consequence of (40) and (41). Proof: Reconsider $\mathcal{H}=\mathcal{F}$ as a function from alter $\mathscr{A}$ into alter $\mathscr{B}$. For every morphisms $f_{1}, f_{2}$ of alter $\mathscr{A}$ such that $f_{1} \triangleright f_{2}$ holds $\mathcal{H}\left(f_{1}\right) \triangleright \mathcal{H}\left(f_{2}\right)$ and $\mathcal{H}\left(f_{1} \circ f_{2}\right)=\mathcal{H}\left(f_{1}\right) \circ \mathcal{H}\left(f_{2}\right)$ by [7, (15), (72), (64)]. For every morphism $f$ of alter $\mathscr{A}$ such that $f$ is identity holds $\mathcal{H}(f)$ is identity by [7, (62)].

(43) Let us consider a non empty category $\mathscr{C}$, morphisms $a_{1}, a_{2}$ of $\operatorname{Alter}(\mathscr{C})$, and morphisms $f_{1}, f_{2}$ of $\mathscr{C}$. Suppose

(i) $a_{1}=f_{1}$, and

(ii) $a_{2}=f_{2}$, and

(iii) $f_{1} \triangleright f_{2}$.

Then $a_{1} \circ a_{2}=f_{1} \circ f_{2}$.

(44) Let us consider a non empty category $\mathscr{C}$, a morphism $f_{1}$ of $\mathscr{C}$, and a morphism $a_{1}$ of Alter $(\mathscr{C})$. Suppose $a_{1}=f_{1}$. Then

(i) $\operatorname{dom} f_{1}=\operatorname{dom} a_{1}$, and

(ii) $\operatorname{cod} f_{1}=\operatorname{cod} a_{1}$.

(45) Let us consider a non empty category $\mathscr{C}$, an object $o_{1}$ of $\mathscr{C}$, and an object $o_{2}$ of Alter $(\mathscr{C})$. If $o_{1}=o_{2}$, then id- $o_{1}=\mathrm{id}_{o_{2}}$. The theorem is a consequence of (22), (24), (44), and (43). Proof: Reconsider $a_{2}=o_{2}$ as a morphism of Alter $(\mathscr{C})$. Reconsider $a_{3}=a_{2}$ as a morphism from $o_{2}$ to $o_{2}$. For every object $b$ of $\operatorname{Alter}(\mathscr{C})$, if $\operatorname{hom}\left(o_{2}, b\right) \neq \emptyset$, then for every morphism $a$ from $o_{2}$ to $b, a \circ a_{3}=a$ and if $\operatorname{hom}\left(b, o_{2}\right) \neq \emptyset$, then for every morphism $a$ from $b$ to $o_{2}, a_{3} \circ a=a$ by [7, (5), (15)].

(46) Let us consider a non empty category $\mathscr{C}$ and a morphism $f$ of $\mathscr{C}$. Then $f$ is identity if and only if there exists an object $o$ of Alter $(\mathscr{C})$ such that $f=\mathrm{id}_{o}$. The theorem is a consequence of (25) and (41).

(47) Let us consider non empty categories $\mathscr{C}, \mathscr{D}$. Then every covariant functor from $\mathscr{C}$ to $\mathscr{D}$ is a functor from $\operatorname{Alter}(\mathscr{C})$ to $\operatorname{Alter}(\mathscr{D})$. The theorem is a consequence of (46), (44), (32), and (45). Proof: Reconsider $\mathcal{H}=\mathcal{F}$ as a function from the carrier' of $\operatorname{Alter}(\mathscr{C})$ into the carrier' of $\operatorname{Alter}(\mathscr{D})$. For every object $a$ of Alter $(\mathscr{C})$, there exists an object $b$ of Alter $(\mathscr{D})$ such that $\mathcal{H}\left(\operatorname{id}_{a}\right)=\operatorname{id}_{b}$. For every morphism $f$ of $\operatorname{Alter}(\mathscr{C}), \mathcal{H}\left(\operatorname{id}_{\operatorname{dom} f}\right)=\operatorname{id}_{\operatorname{dom}(\mathcal{H}(f))}$ and $\mathcal{H}\left(\operatorname{id}_{\operatorname{cod} f}\right)=\operatorname{id}_{\operatorname{cod}(\mathcal{H}(f))}$. For every morphisms $f, g$ of $\operatorname{Alter}(\mathscr{C})$ such that $\operatorname{dom} g=\operatorname{cod} f$ holds $\mathcal{H}(g \circ f)=\mathcal{H}(g) \circ \mathcal{H}(f)$ by [7, (15), (16)].

(48) Let us consider object-categories $\mathscr{C}, \mathscr{D}$. Then every covariant functor from alter $\mathscr{C}$ to alter $\mathscr{D}$ is a functor from $\mathscr{C}$ to $\mathscr{D}$. The theorem is a consequence of (41), (26), and (27). Proof: Reconsider $\mathcal{H}=\mathcal{F}$ as a function from the carrier' of $\mathscr{C}$ into the carrier' of $\mathscr{D}$. For every object $a$ of $\mathscr{C}$, there 
exists an object $b$ of $\mathscr{D}$ such that $\mathcal{H}\left(\mathrm{id}_{a}\right)=\operatorname{id}_{b}$. For every morphism $f$ of $\mathscr{C}$, $\mathcal{H}\left(\operatorname{id}_{\operatorname{dom} f}\right)=\operatorname{id}_{\operatorname{dom}(\mathcal{H}(f))}$ and $\mathcal{H}\left(\operatorname{id}_{\operatorname{cod} f}\right)=\operatorname{id}_{\operatorname{cod}(\mathcal{H}(f))}$ by [7, (15)]. For every morphisms $f, g$ of $\mathscr{C}$ such that $\operatorname{dom} g=\operatorname{cod} f$ holds $\mathcal{H}(g \circ f)=\mathcal{H}(g) \circ \mathcal{H}(f)$ by [7, (15), (16)].

Let us consider object-categories $\mathscr{C}_{1}, \mathscr{C}_{2}$. Now we state the propositions:

(49) If alter $\mathscr{C}_{1} \cong$ alter $\mathscr{C}_{2}$, then $\mathscr{C}_{1} \cong \mathscr{C}_{2}$.

(50) Suppose the carrier' of $\mathscr{C}_{1}=$ the carrier' of $\mathscr{C}_{2}$ and the composition of $\mathscr{C}_{1}=$ the composition of $\mathscr{C}_{2}$. Then $\mathscr{C}_{1} \cong \mathscr{C}_{2}$.

Now we state the propositions:

(51) Let us consider an object-category $\mathscr{C}$. Then $\mathscr{C} \cong$ Alter $($ alter $\mathscr{C})$.

(52) Let us consider a non empty category $\mathscr{C}$. Then $\mathscr{C} \cong$ alter $\operatorname{Alter}(\mathscr{C})$. The theorem is a consequence of (16) and (18). Proof: Set $\mathscr{D}=\operatorname{alter} \operatorname{Alter}(\mathscr{C})$. Reconsider $\mathcal{F}=\operatorname{id}_{\mathscr{C}}$ as a functor from $\mathscr{C}$ to $\mathscr{D}$. Reconsider $\mathcal{G}=\operatorname{id}_{\mathscr{C}}$ as a functor from $\mathscr{D}$ to $\mathscr{C}$. For every morphism $f$ of $\mathscr{C}$ such that $f$ is identity holds $\mathcal{F}(f)$ is identity. For every morphisms $f_{1}, f_{2}$ of $\mathscr{C}$ such that $f_{1} \triangleright f_{2}$ holds $\mathcal{F}\left(f_{1}\right) \triangleright \mathcal{F}\left(f_{2}\right)$ and $\mathcal{F}\left(f_{1} \circ f_{2}\right)=\mathcal{F}\left(f_{1}\right) \circ \mathcal{F}\left(f_{2}\right)$. For every morphism $f$ of $\mathscr{D}$ such that $f$ is identity holds $\mathcal{G}(f)$ is identity. For every morphisms $f_{1}$, $f_{2}$ of $\mathscr{D}$ such that $f_{1} \triangleright f_{2}$ holds $\mathcal{G}\left(f_{1}\right) \triangleright \mathcal{G}\left(f_{2}\right)$ and $\mathcal{G}\left(f_{1} \circ f_{2}\right)=\mathcal{G}\left(f_{1}\right) \circ \mathcal{G}\left(f_{2}\right)$.

\section{REFERENCES}

[1] Jiri Adamek, Horst Herrlich, and George E. Strecker. Abstract and Concrete Categories: The Joy of Cats. Dover Publication, New York, 2009.

[2] Grzegorz Bancerek. Cardinal numbers Formalized Mathematics, 1(2):377-382, 1990.

[3] Grzegorz Bancerek. The ordinal numbers. Formalized Mathematics, 1(1):91-96, 1990.

[4] Grzegorz Bancerek and Krzysztof Hryniewiecki. Segments of natural numbers and finite sequences. Formalized Mathematics, 1(1):107-114, 1990.

[5] Francis Borceaux. Handbook of Categorical Algebra I. Basic Category Theory, volume 50 of Encyclopedia of Mathematics and its Applications. Cambridge University Press, Cambridge, 1994.

[6] Czesław Byliński. Binary operations Formalized Mathematics, 1(1):175-180, 1990.

[7] Czesław Byliński. Introduction to categories and functors Formalized Mathematics, 1 (2):409-420, 1990.

[8] Czesław Byliński. Subcategories and products of categories. Formalized Mathematics, 1 (4):725-732, 1990.

[9] Czesław Byliński. Functions and their basic properties Formalized Mathematics, 1(1): 55-65, 1990.

[10] Czesław Byliński. Functions from a set to a set Formalized Mathematics, 1(1):153-164, 1990.

[11] Czesław Byliński. The modification of a function by a function and the iteration of the composition of a function. Formalized Mathematics, 1(3):521-527, 1990.

[12] Czesław Byliński. Partial functions Formalized Mathematics, 1(2):357-367, 1990.

[13] Czesław Byliński. Some basic properties of sets Formalized Mathematics, 1(1):47-53, 1990.

[14] Agata Darmochwał. Finite sets Formalized Mathematics, 1(1):165-167, 1990.

[15] Krzysztof Hryniewiecki. Graphs. Formalized Mathematics, 2(3):365-370, 1991. 
[16] Saunders Mac Lane. Categories for the Working Mathematician, volume 5 of Graduate Texts in Mathematics. Springer Verlag, New York, Heidelberg, Berlin, 1971.

[17] Beata Padlewska. Families of sets. Formalized Mathematics, 1(1):147-152, 1990.

[18] Andrzej Trybulec. Categories without uniqueness of cod and dom Formalized Mathematics, 5(2):259-267, 1996.

[19] Andrzej Trybulec. Isomorphisms of categories. Formalized Mathematics, 2(5):629-634, 1991.

[20] Zinaida Trybulec. Properties of subsets. Formalized Mathematics, 1(1):67-71, 1990.

[21] Edmund Woronowicz. Relations and their basic properties Formalized Mathematics, 1 (1):73-83, 1990.

[22] Edmund Woronowicz. Relations defined on sets Formalized Mathematics, 1(1):181-186, 1990.

Received October 7, 2013 